\title{
Ayn Soukhna (2020)
}

Mahmoud Abd el-Raziq et Claire Somaglino

\section{OpenEdition \\ Journals}

Édition électronique

URL : https://journals.openedition.org/baefe/2665

DOI : 10.4000/baefe.2665

ISSN : 2732-687X

Éditeur

ResEFE

\section{Référence électronique}

Mahmoud Abd el-Raziq, Claire Somaglino, « Ayn Soukhna (2020) » [notice archéologique], Bulletin archéologique des Écoles françaises à l'étranger [En ligne], Égypte, mis en ligne le 30 mai 2021, consulté le 27 juillet 2021. URL : http://journals.openedition.org/baefe/2665; DOI : https://doi.org/10.4000/ baefe. 2665

Ce document a été généré automatiquement le 27 juillet 2021.

\section{cc) (†) $\odot$}

Le Bulletin archéologique des Écoles françaises à l'étranger est mise à disposition selon les termes de la Licence Creative Commons Attribution - Pas d'Utilisation Commerciale - Pas de Modification 4.0 International. 


\title{
Ayn Soukhna (2020)
}

\author{
Mahmoud Abd el-Raziq et Claire Somaglino
}

\section{NOTE DE L'AUTEUR}

Année de la campagne : 2020 ( 4 janvier - 9 février)

Autorité nationale présente : Le ministère du Tourisme et des Antiquités (MoTA) était représenté par Marwa Salah el-Din, inspectrice du S.A. de Suez

Numéro et intitulé de l'opération de terrain : 17131 - Ayn Soukhna

Composition de l'équipe de terrain : L'équipe était composée de Mahmoud Abd el-

Raziq (égyptologue, université du canal de Suez), Claire Somaglino (égyptologue, Sorbonne Université), Pierre Tallet (égyptologue, Sorbonne Université), Georges Castel (archéologie, architecte, Ifao), Adeline Bats (égyptologue), Georges Verly (archéométallurgiste), Claire Malleson (archéobotaniste, American University in Beirut), Abeid Mahmoud (restaurateur, Ifao), Ihab Mohamed (photographe, Ifao), Sara Zaia (archéologue, Harvard University), Mohamed Gaber (topographe, Ifao) et Adel Farouq (intendant, CSA). Les ouvriers étaient placés sous la direction du raïs Gamal Nasr el-Din.

Partenariats institutionnels : Cette mission a pu être réalisée grâce au support de la Fondation Honor Frost, ainsi que de Sorbonne Université et du Centre national de la recherche scientifique (CNRS, UMR 8167 Orient et Méditerranée, équipe Mondes pharaoniques).

La fouille de la zone basse du site (zone 1) [pour le plan de position des secteurs mentionnés ci-dessous, voir fig. 1 du rapport Ayn Soukhna 2019, https:// journals.openedition.org/baefe/1083]

\section{La fouille archéologique}

1 L'exploration systématique de la zone du site localisée en contrebas du piémont montagneux s'est poursuivie au cours de la saison 2020. La zone 1 est occupée, à l'Ancien Empire et au début du Moyen Empire, par des installations portuaires, de 
l'habitat et des structures artisanales, édifiées par les expéditions égyptiennes qui se rendaient dans la zone minière du Sud-Sinaï pour y exploiter mines de cuivre et de turquoise. La fouille extensive et minutieuse de ces installations permet de reconstituer le quotidien des équipes qui évoluaient sur le site durant le temps d'une expédition ainsi que de mieux comprendre l'organisation logistique de ces entreprises commanditées par le pouvoir royal. Ayn Soukhna constitue également un site de référence pour l'histoire des techniques, en particulier pour la métallurgie du cuivre au Moyen Empire. Cette question de la reconstitution des chaînes opératoires est donc également essentielle dans le déploiement de nos travaux.

2 Nous avons tout d'abord poursuivi la fouille dans les secteurs voisins S6-Nord et Kôm 14-Ouest.

3 En S6-Nord, la fouille des derniers niveaux préservés datant du début du Moyen Empire a été achevée. Une cache de céramiques dans une fosse y a été dégagée (fig. 1). Ce type de cache est régulièrement retrouvé sur le site : les équipes égyptiennes, à la fin d'une expédition, stockaient des lots d'objets utilitaires, généralement céramiques et outils en pierre, dans les angles de pièces à l'intérieur des bâtiments ou dans des fosses qu'ils recouvraient. L'objectif était vraisemblablement de retrouver ce matériel lors de l'expédition suivante. L'ensablement du site ou encore le temps qui pouvait s'écouler entre deux expéditions faisait que toutes les caches n'étaient pas retrouvées.

4 La fosse dégagée cette année contenait cinq jarres de stockage de céréales en Marl-C, une petite jarre, deux bols, une cruche à décor en peigne peint en rouge et un vase à bière fragmentaire. Le tout était soigneusement rangé dans la fosse grossièrement creusée dans le sol: les jarres étaient calées les unes contre les autres et les bols empilés puis placés sur le col d'une jarre. Un lot de 14 broyons hémisphériques en grès local avait également été déposé, une fois les céramiques partiellement enterrées dans la fosse. La plupart des céramiques présentaient la même marque non hiéroglyphique, gravée après cuisson sur la panse de la jarre ou du bol. C'est un phénomène bien connu à Ayn Soukhna au début du Moyen Empire et qui signalait l'appartenance de l'objet et son éventuel contenu à l'une des équipes de l'expédition. 
Fig. 1. Cachette de céramiques et d'outils en pierre du début du Moyen Empire dans le secteur S6Nord, vue est-ouest.

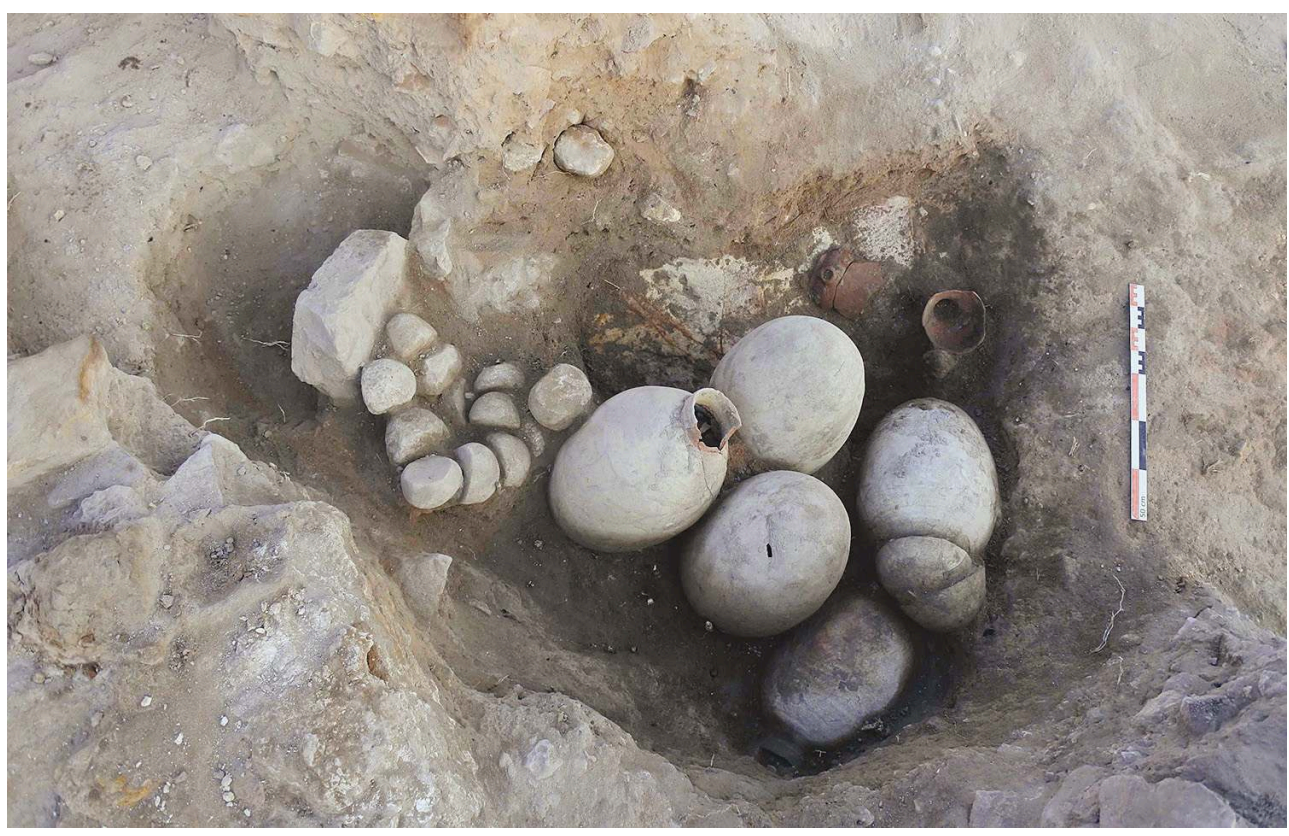

(C) Mission archéologique d'Ayn Soukhna. 17131_2020_NDMPF_001

5 Cette fosse du début du Moyen Empire a coupé les vestiges d'occupation datant de l'Ancien Empire situés à cet endroit. Le long du promontoire rocheux qui délimite l'occupation de la zone basse au sud, une nouvelle pièce de cette période a en effet été identifiée. Comme ailleurs, le rocher contre lequel elle s'appuie a été aménagé (régularisation du rocher, fosses, caches). Au nord de la pièce, on observe un espace ouvert avec des zones de foyer pour la cuisson des aliments et des zones de rejet où de nombreux moules-bedja et plats à pain ont été retrouvés. D'autres espaces similaires avaient été identifiés plus au nord du secteur 56 -Nord les années précédentes; ils datent de la seconde moitié de la IV ${ }^{e}$ dynastie pour les plus anciens, c'est-à-dire de la première occupation connue du site durant l'Ancien Empire. Nous avons d'ailleurs cette année dégagé la suite d'un important dépôt de coquillages, en partie fouillé en 2019 et datant de cette phase d'occupation du site. L'essentiel du dépôt était constitué de Nerita sanguinolenta Menke, 1829 (nombre minimum d'individus : 2047 ). La proximité des foyers laisse penser que ces mollusques étaient consommés par les équipes égyptiennes.

6 Toujours près du promontoire rocheux, dans les niveaux datant de la $\mathrm{V}^{\mathrm{e}}$ dynastie, l'élargissement de la zone étudiée a permis de dégager le reste de l'aire de préparation de l'argile identifiée en 2019. Il s'agissait à cet endroit de transformer l'argile locale, récoltée dans les ouadis avoisinants, pour en faire des bouchons de jarre. Plusieurs fosses de petites dimensions, peu profondes, servaient à mixer l'argile. Autour, se trouvaient des mottes d'argile plus ou moins raffinées. Ensuite, cette matière était préformée en boudins sub-rectangulaires d'une dizaine de centimètres de long, standardisés, qui étaient empilés en attendant d'être utilisés pour obturer une jarre. Plusieurs bouchons de jarre usagés ont d'ailleurs été retrouvés dans la zone, montrant que cette activité était liée à une zone de préparation alimentaire située à proximité, et qui avait été fouillée lors de la mission 2019. 
7 Dans le secteur adjacent, Kôm 14-ouest, s'est également poursuivie la fouille des niveaux archéologiques de l'Ancien Empire (fig. 2). Nous avons pu, sur l'essentiel de la superficie étudiée, atteindre la première phase d'occupation, caractérisée là encore par de nombreux foyers et des lots de moules et plats à pain. L'essentiel du matériel archéologique, comme en S6-Nord, était composé d'outils en pierre et en silex, et de céramique.

Fig. 2. Secteurs Kôm 14-ouest (à gauche) et S6-Nord (à droite) à la fin de la mission 2020, vue nord-ouest à sud-est.

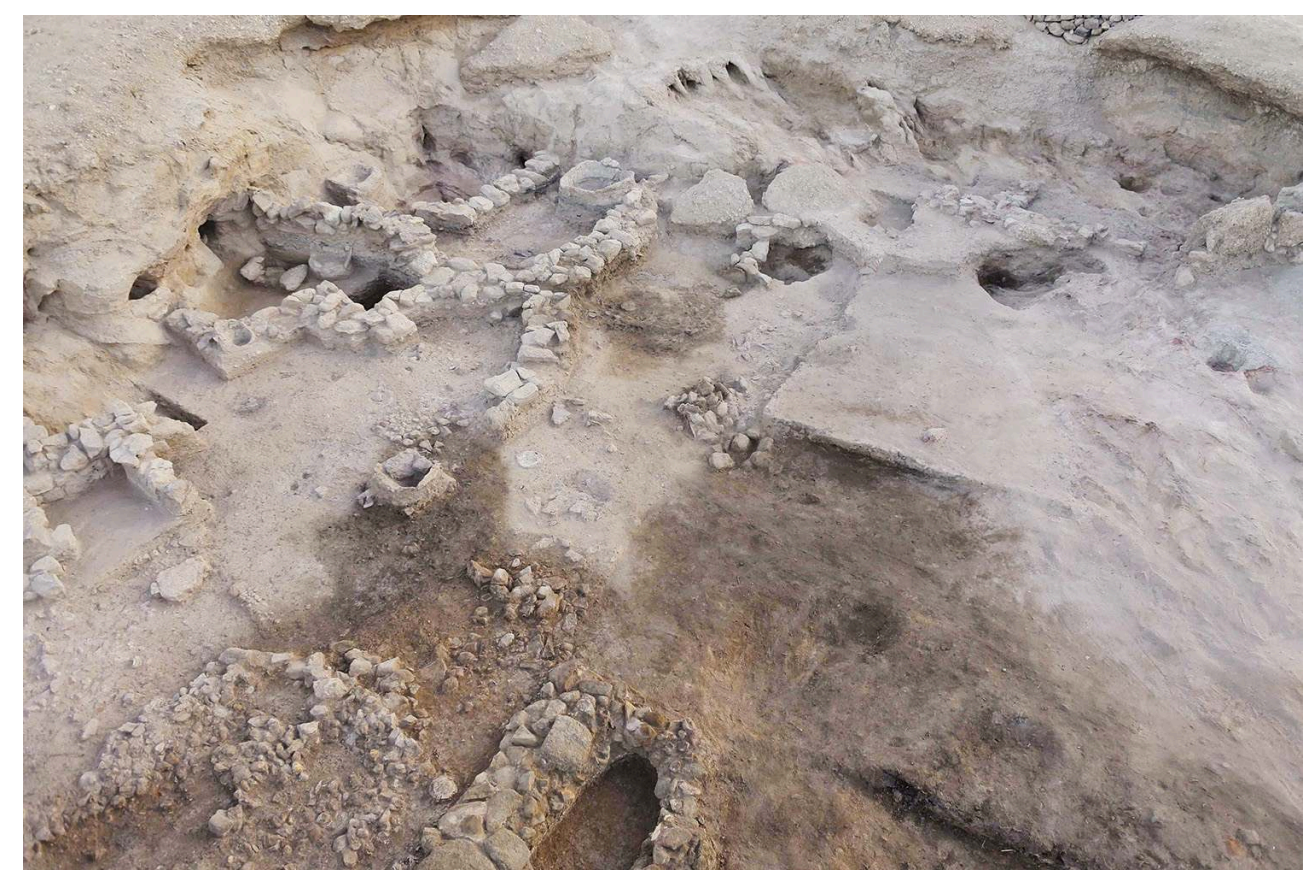

(C) Mission archéologique d'Ayn Soukhna. 17131_2020_NDMPF_002

La fouille du secteur situé au nord du Kôm 14 a été plus largement ouverte cette année $(12 \times 7 \mathrm{~m})$, après la découverte lors de la mission 2019 de vestiges du Moyen Empire et de l'Ancien Empire dans un sondage. Il s'agissait de mieux comprendre en particulier le contexte de la grande fosse du Kôm 14 creusée à l'Ancien Empire dans le substrat rocheux pour aider à l'entretien et au remontage des navires.

L'élargissement du sondage a permis d'identifier un bâtiment en pierres sèches datant du début du Moyen Empire (4,6 par 2,5 m) et une petite chambre chaude dédiée à la cuisson du pain, datant de la même époque (fig. 3). Les niveaux sous-jacents datent du début du Moyen Empire et de l'Ancien Empire. Aucune structure n'y a été identifiée, mais seulement des rejets de foyers et une faible quantité de céramique. Un deuxième sondage de $3 \times 3 \mathrm{~m}$ a donc été réalisé plus au nord, mais n'a livré qu'une faible quantité de rejets de l'Ancien Empire. Il paraît donc probable que cette bande centrale corresponde au début du Moyen Empire, comme dans le secteur S41-Est, à une zone ouverte entre deux îlots de construction. Des sondages datant du début de la fouille du site, au nord, près de la route, avaient en effet révélé des traces de bâtiments de cette époque. Pour l'Ancien Empire, les bâtiments et installations artisanales semblent donc situés uniquement le long de l'escarpement rocheux. Il n'est pas à exclure cependant que d'autres constructions liées à l'activité portuaire aient pu être édifiées directement 
sur le littoral situé à quelque $200 \mathrm{~m}$ plus au nord (emplacement aujourd'hui occupé par une route et un hôtel).

Fig. 3. Secteur Kôm 14-Nord. Bâtiment du début du Moyen Empire, vue nord-sud.

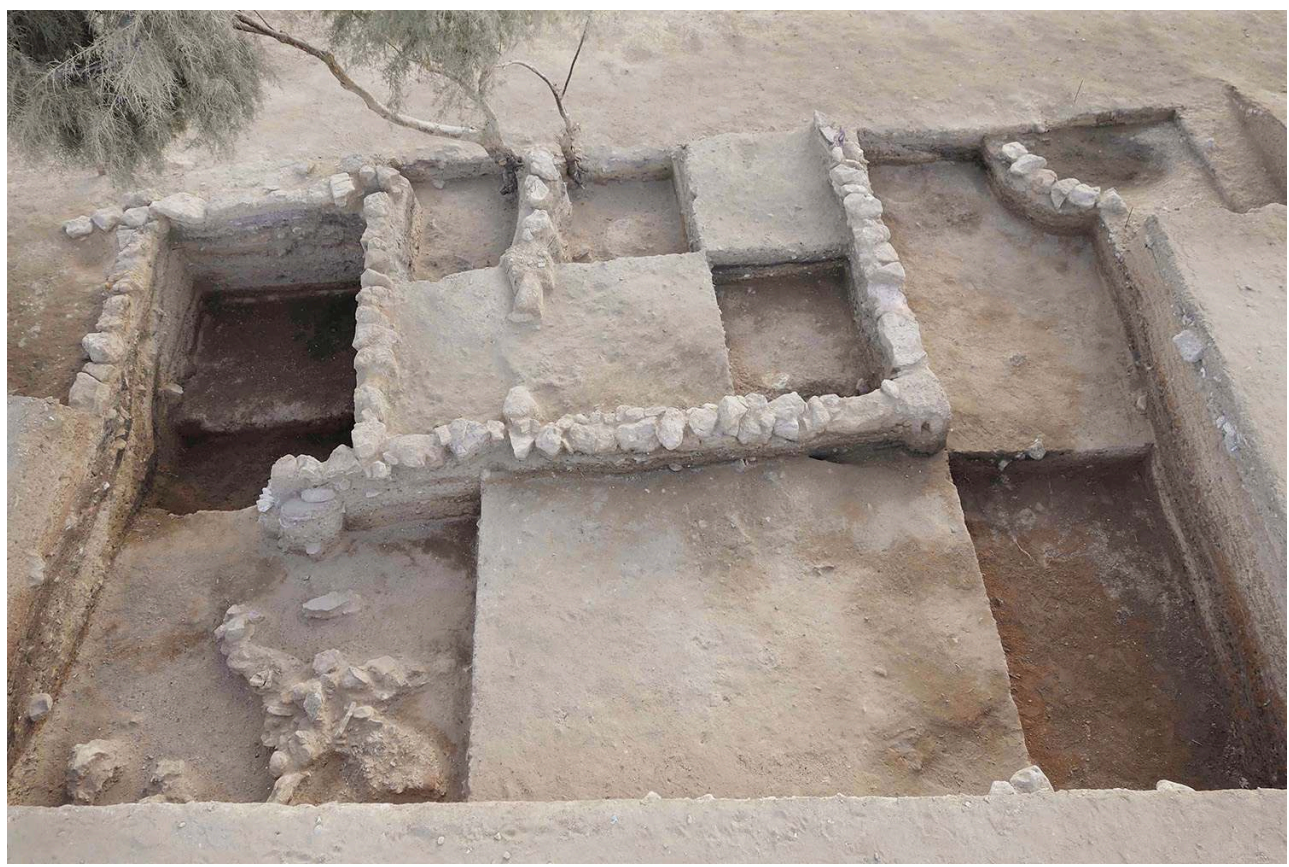

(c) Mission archéologique d'Ayn Soukhna. 17131_2020_NDMPF_003

Fig. 4. Secteur S39, à l'ouest de la zone 1, à la fin de la saison 2020 (vue ouest-est). On note à droite l'atelier de métallurgie proprement dit avec ses quatre fours de réduction du minerai de cuivre et à gauche la construction en briques crues.

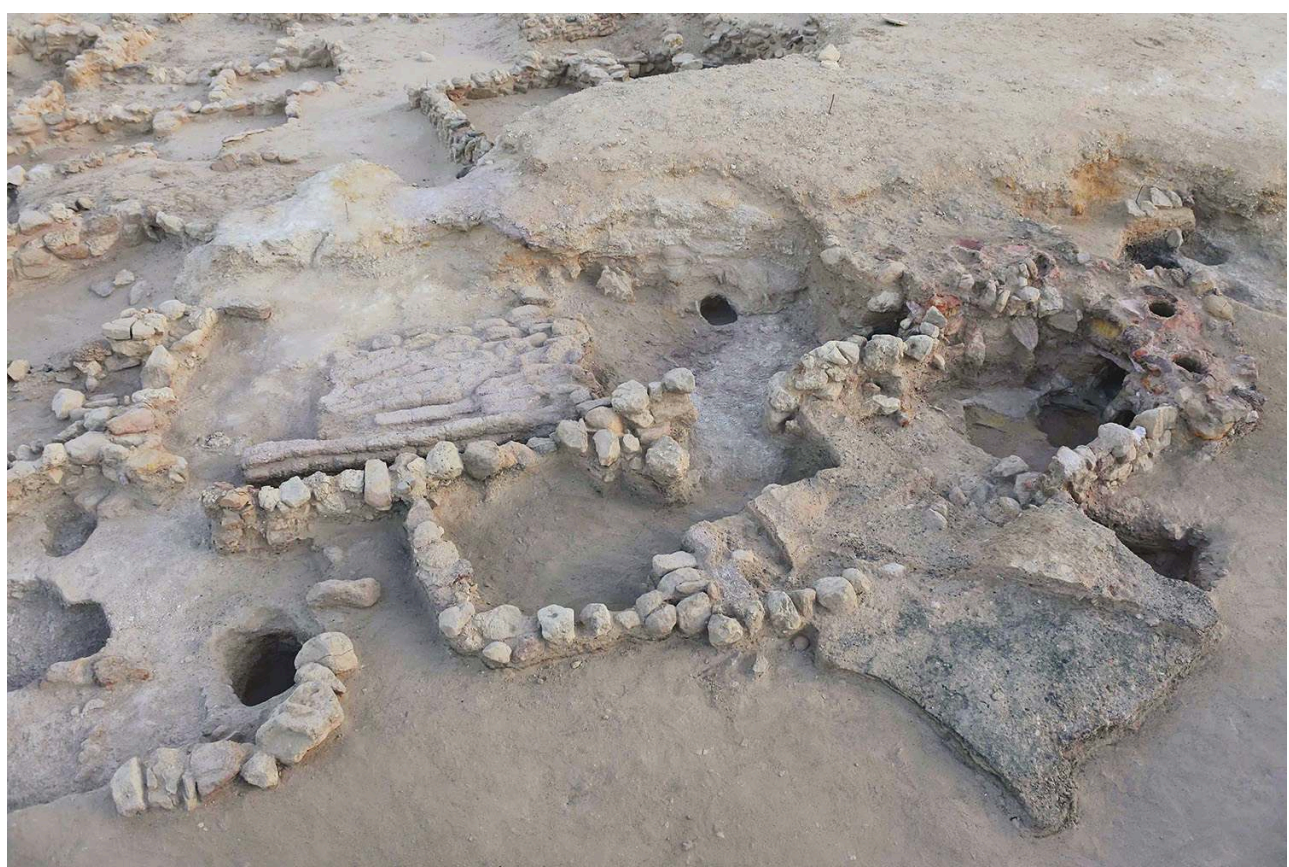

(c) Mission archéologique d'Ayn Soukhna. 17131_2020_NDMPF_004 
La fouille de la partie centrale et orientale de la zone 1 étant presque achevée, c'est vers l'ouest que nous reportons progressivement nos travaux depuis deux ans. L'étude du secteur S39 a ainsi été poursuivie et agrandie cette année (fig. 4). Un atelier de métallurgie du cuivre du début du Moyen Empire y a été progressivement dégagé depuis l'an dernier, avec notamment une batterie de quatre fours de réduction adossée au promontoire rocheux. D'autres bâtiments avaient également été construits en plusieurs phases à proximité, toujours appuyés sur le rocher. Lors du nettoyage des murs de pierre sèche de cet ensemble de pièces, une petite amulette en calcaire a été retrouvée. Elle a la forme d'une stèle cintrée miniature, ou du plat d'un grand sceau administratif, et présente la gravure d'un singe entouré d'éléments géométriques et végétaux.

11 Une construction en briques crues - ce qui est rare sur le site - a continué à être fouillée. Elle semble appartenir à la première phase de construction du début du Moyen Empire. On peut encore discerner l'arase d'un mur à l'ouest et les vestiges d'un pavement de briques. Le matériel qui y a été retrouvé est cependant trop peu abondant pour aider à comprendre la fonction précise de cet espace.

12 Nous avons poursuivi la fouille d'un épais niveau de rejets cendreux au sud de S39. Il s'agit d'une partie des rejets des fours de l'atelier de réduction du cuivre, comportant de nombreux déchets métallurgiques et outils de pierre. La fouille fine de l'ensemble de cet atelier et de ses environs depuis l'an dernier a permis d'affiner considérablement notre compréhension des techniques de tri à l'ouverture des fours de réduction, ainsi que d'appréhender la question de la gestion des déchets.

Sous les premiers bâtiments du Moyen Empire du secteur S39, plusieurs fosses de forme ovale ont été retrouvées cette année. Elles sont directement creusées dans le substrat rocheux et pourraient dater de l'Ancien Empire. En effet, une série de fosses et foyers, cette fois-ci clairement datée de l'Ancien Empire par la céramique qui y est associée, a été identifiée au sud de l'atelier de métallurgie, le long de la pente du gebel (fig. 5). Plusieurs de ces fosses ont servi de foyers, alors que d'autres étaient tapissées de pierres plates et tessons de céramique, le tout recouvert de mouna, sans aucune trace de combustion (fig. 6). Nous avions déjà trouvé deux aménagements similaires l'an dernier dans le secteur Kôm 14-ouest, dans les niveaux datant de la $\mathrm{V}^{\mathrm{e}}$ dynastie. Il s'agit de la première occurrence de vestiges datant de l'Ancien Empire dans la zone ouest de la partie basse. 
Fig. 5. Fosses et aménagements de l'Ancien Empire, réalisés directement sur la pente du promontoire rocheux, au sud du secteur S39, vue ouest-est.

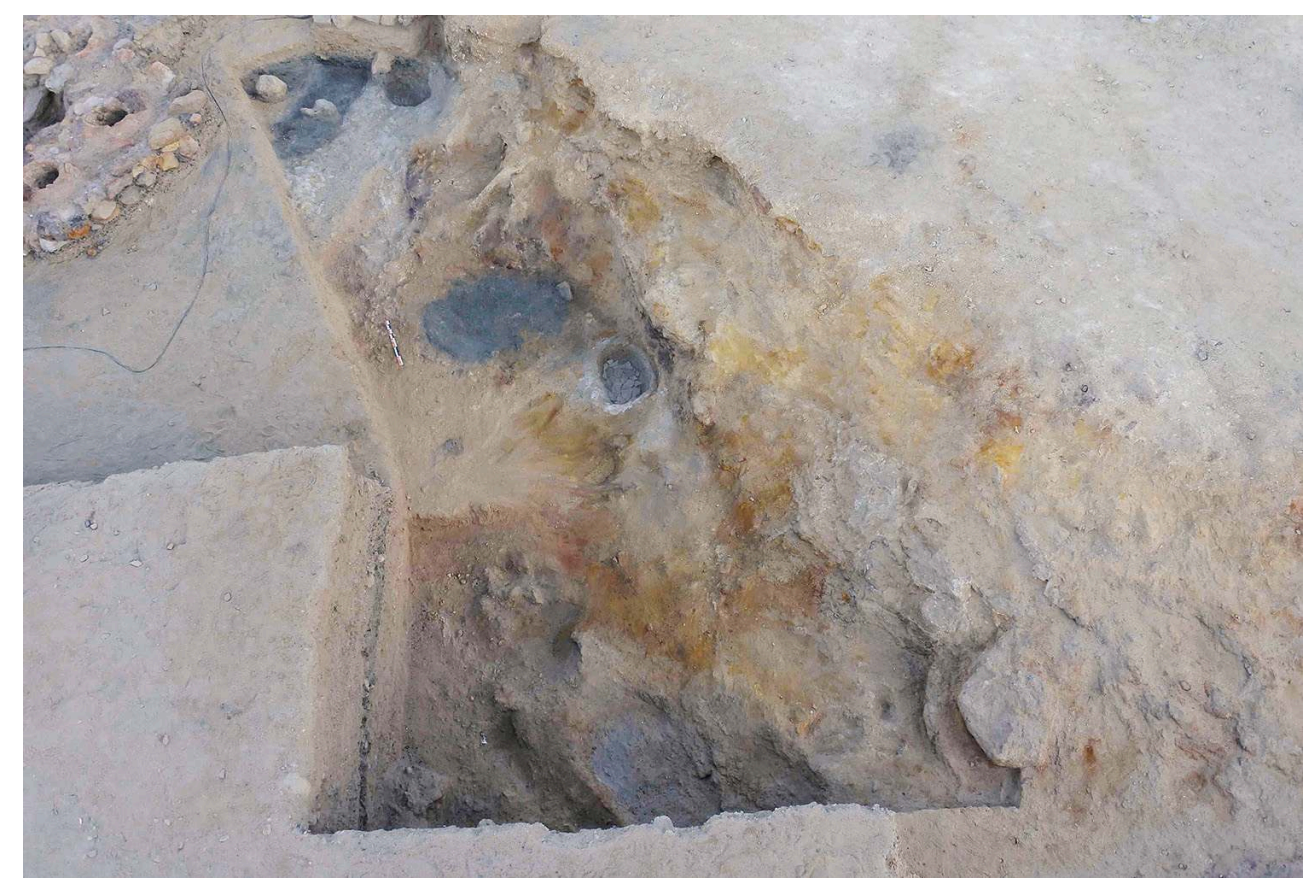

(c) Mission archéologique d'Ayn Soukhna. 17131_2020_NDMPF_005

Fig. 6. Petite fosse aménagée dans la pente du gebel et datant de l'Ancien Empire. Secteur S39.

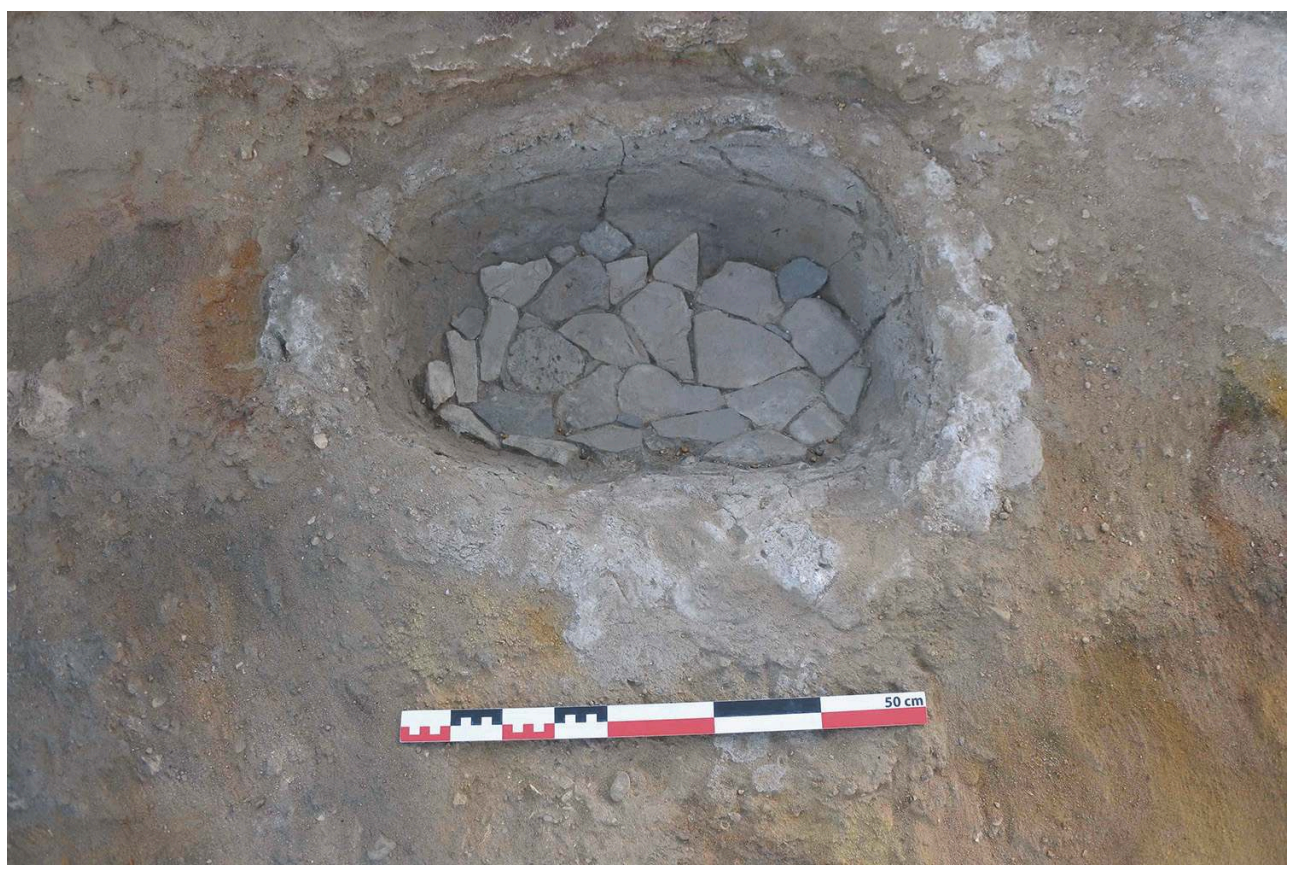

(C Mission archéologique d'Ayn Soukhna. 17131_2020_NDMPF_006

\section{Autres activités}

- Georges Verly a poursuivi l'étude de la métallurgie du cuivre sur le site, tout particulièrement tournée vers les vestiges du début du Moyen Empire. Il a analysé et 
documenté (relevés 3D) l'ensemble des fours de réduction fouillés depuis 2019 dans le secteur S39, ainsi que les rejets tout autour de l'atelier, afin d'affiner la compréhension de la chaîne opératoire.

- Adeline Bats a poursuivi son étude des jarres de stockage en Marl-C du début du Moyen Empire ainsi que du système de marque avant et après-cuisson qui sont gravées sur ces céramiques (dessin des céramiques, enregistrement des marques dans une base de données spécifique). Elle a également continué l'étude des moules à pain de l'Ancien et du Moyen Empire, que l'on retrouve en nombre sur le site.

- Claire Malleson a étudié les restes archéobotaniques sous microscope, après flottation et tamisage. La majorité des échantillons analysés provient des foyers de l'Ancien Empire des secteurs Kôm 14-ouest et S6-Nord.

\section{Publications}

- Frederik W. Rademakers, Georges Verly, Claire Somaglino, Patrick Degryse, « Geochemical Changes During Egyptian Copper Smelting? An Experimental Approach to the Ayn Soukhna Process and Broader Implications for Archaeometallurgy », JAS 122, 2020, article en ligne, https://doi.org/10.1016/j.jas.2020.105223, consulté le 5 avril 2021.

- Adeline Bats, «The Production of Bread in Conical Moulds at the Beginning of the Egyptian Middle Kingdom. The Contribution of Experimental Archaeology », Journal of Archaeological Science Reports 34(A), 2020, article en ligne, https://doi.org/10.1016/j.jasrep.2020.102631, consulté le 5 avril 2021.

\section{Communications scientifiques}

- Claire Somaglino, « Dernières nouvelles des fouilles du port pharaonique d'Ayn Soukhna en mer Rouge », 19 janvier 2020, Ifao, https://www.youtube.com/watch?v=DcL6o5LWPmg, consulté le 5 avril 2021.

- Claire Somaglino, «Ayn Soukhna, port et plateforme logistique au cœur du réseau pharaonique en mer Rouge », université d'Artois, journée d'étude "L'appel du large. II. Fleuves et mers dans le monde gréco-romain ", mars 2020, organisée par A. Gagey, M.O. Laforge, S. Lebreton et P. Schneider. 


\section{INDEX}

\section{Thèmes : IFAO}

Année de l'opération : 2020

chronologie https://ark.frantiq.fr/ark:/26678/pcrtPezBqzEcKR, https://ark.frantiq.fr/ark:/ 26678/pcrtQfmmy4haAf

lieux https://ark.frantiq.fr/ark:/26678/pcrt1iOWfobZgH, https://ark.frantiq.fr/ark:/26678/ pcrtEo791eXy7G

nature https://ark.frantiq.fr/ark:/26678/pcrtb1E0Dz7cSX

sujets 26678/pcrt9PJh9aTXv4, https://ark.frantiq.fr/ark:/26678/pcrtwh5icnwutJ, https://

ark.frantiq.fr/ark:/26678/pcrtItMnfHySi0, 26678/pcrtvWHPMsEEqO, https://ark.frantiq.fr/ark:/ 26678/pcrtmt7DWlRknS, https://ark.frantiq.fr/ark:/26678/pcrt16pnvNar4g

\section{AUTEURS}

\section{MAHMOUD ABD EL-RAZIQ}

Égyptologue, université du canal de Suez

\section{CLAIRE SOMAGLINO}

Égyptologue, Sorbonne Université 